02

\title{
Анализ экспериментальных результатов по модели Гавриляка-Негами в диэлектрической спектроскопии
}

\author{
(ㄱ А.С. Волков, Г.Д. Копосов, Р.О. Перфиильев, А.В. Тягунин \\ Северный (Арктический) фредеральный университет им. М.В. Ломоносова, \\ 163002 Архангельск, Россия \\ e-mail: a.s.volkov@narfu.ru
}

Поступила в редакцию 11.09.2017 г.

Рассмотрена проблема идентификации экспериментальных результатов по исследованию частотных зависимостей действительной и мнимой частей диэлектрической проницаемости на предмет соответствия одной из признанных моделей частотной дисперсии: модели Дебая, Коул-Коула, Дэвидсона-Коула и Гавриляка-Негами. На основе выражений для компонент комплексной диэлектрической проницаемости с использованием математического анализа разработана последовательность шагов по определению характеристических параметров обобщенной модели Гавриляка-Негами: статической $\varepsilon_{S}$ и высокочастотной $\varepsilon_{\infty}$ диэлектрической проницаемости, показателей частотной дисперсии $\alpha$ и $\beta$, времени релаксации $\tau$. Приведен пример расчета параметров $\varepsilon_{S}, \varepsilon_{\infty}, \alpha, \beta$ и $\tau$ для образца мерзлой дисперсной среды на основе порошка мелкозернистого кварца при влажности $13 \%$ в диапазоне температур от -140 до $0{ }^{\circ} \mathrm{C}$.

DOI: $10.21883 /$ OS.2018.02.45525.200-17

\section{Введение}

Одной из проблем физики диэлектриков является установление вида частотной зависимости действительной и мнимой частей комплексной диэлектрической проницаемости $\varepsilon^{*}=\varepsilon^{\prime}-j \varepsilon^{\prime \prime}$. Вынужденные колебания в переменном электрическом поле зависят от того, имеет ли вещество частицы с независящей от температуры собственной частотой колебаний или вынужденные колебания подвержены релаксационному температурному процессу с некоторым временем релаксации. Поляризация льда в мерзлых влагосодержащих дисперсных средах, изучением которой занимается Лаборатория физики дисперсных систем САФУ имени М.В. Ломоносова, является релаксационной.

Первая теория частотной зависимости диэлектрической проницаемости полярных диэлектриков была создана Дебаем в 1929 г. [1]:

$$
\varepsilon=\varepsilon_{\infty}+\frac{\varepsilon_{S}-\varepsilon_{\infty}}{1+i \omega \tau}
$$

В 1941 г. Коулы [2] предложили несколько иную математическую модель диэлектрической релаксации:

$$
\varepsilon=\varepsilon_{\infty}+\frac{\varepsilon_{S}-\varepsilon_{\infty}}{1+(i \omega \tau)^{1-\alpha}} .
$$

Коул и Дэвидсон [3] в 1951 г. видоизменили дисперсионную формулу, приведя ее к виду

$$
\varepsilon=\varepsilon_{\infty}+\frac{\varepsilon_{S}-\varepsilon_{\infty}}{(1+i \omega \tau)^{\beta}} .
$$

В 1966 г. Гавриляк и Негами [4] продолжили развитие математической модели, предложив следующее дисперсионное соотношение:

$$
\varepsilon=\varepsilon_{\infty}+\frac{\varepsilon_{S}-\varepsilon_{\infty}}{\left(1+(i \omega \tau)^{1-\alpha}\right)^{\beta}} .
$$

Модель Гавриляка-Негами (4) можно рассматривать как обобщение предыдущих моделей частотной дисперсии:

- при $\alpha=0$ и $\beta=1$ - модель Дебая;

- при $1>\alpha>0$ и $\beta=1-$ модель Коул-Коула;

- при $\alpha=0$ и $1>\beta>0$ - модель Дэвидсона-Коула.

В этой модели компоненты комплексной диэлектрической проницаемости находятся [5]:

$$
\begin{gathered}
\varepsilon^{\prime}=\varepsilon_{\infty}+\left(\varepsilon_{S}-\varepsilon_{\infty}\right) r^{-\beta / 2} \cos \beta \theta, \\
\varepsilon^{\prime \prime}=\left(\varepsilon_{S}-\varepsilon_{\infty}\right) r^{-\beta / 2} \sin \beta \theta,
\end{gathered}
$$

где

$$
\begin{gathered}
r=\left[1+(\omega \tau)^{1-\alpha} \sin \left(\frac{\alpha \pi}{2}\right)\right]^{2}+\left[(\omega \tau)^{1-\alpha} \cos \left(\frac{\alpha \pi}{2}\right)\right]^{2} \\
\theta=\operatorname{arctg}\left[\frac{(\omega \tau)^{1-\alpha} \cos \left(\frac{\alpha \pi}{2}\right)}{1+(\omega \tau)^{1-\alpha} \sin \left(\frac{\alpha \pi}{2}\right)}\right] .
\end{gathered}
$$

Попытка определить параметры частотной дисперсии $\alpha, \beta$ и $\tau$ предпринимались и ранее. Лукичев [6] видоизменил математическую форму частотной дисперсии Гавриляка-Негами, приведя ее к не вполне характерному виду, использовал при анализе не совсем правильное соотношение $\omega\left(\varepsilon_{\max }^{\prime \prime}\right) \tau=1$. Кроме этого, представляется нелогичным введение параметра $C=\frac{\log \left(\tau_{0}\right)^{C}}{\log \left(\tau_{0}\right)}$, где $\log \left(\tau_{0}\right)$ выбирается исходя из начальной температуры эксперимента.

Богатин с соавторами [7] предложили метод экстраполяции для определения вида распределения релаксаторов в диэлектриках. Однако приведенные уравнения для $\varepsilon^{\prime}$ и $\varepsilon^{\prime \prime}$ при $\omega \rightarrow \infty$ не совсем согласуются с выражениями $\varepsilon^{\prime}$ и $\mathcal{\varepsilon}^{\prime \prime}$ по модели ГаврилякаНегами.

Учитывая сказанное выше, был проведен математический анализ дисперсионных соотношений и выработан 
экспериментально ориентированный алгоритм для определения диэлектрической частотной дисперсии.

\section{Алгоритм расчета}

Шаг 1 выработанного алгоритма предполагает построение диаграмм Арганда (Коул-Коула), представляющих зависимость релаксационной части $\varepsilon_{r e l}^{\prime \prime}=\varepsilon^{\prime \prime}-\frac{\sigma_{S}}{\varepsilon_{0} \omega}$ от действительной части $\varepsilon^{\prime}$. В предыдущем уравнении $\sigma_{S}-$ статическая удельная электрическая проводимость, $\varepsilon_{0}$ - диэлектрическая постоянная. Полиномиальное представление этой зависимости позволяет при $\varepsilon_{\text {rel }}^{\prime \prime} \rightarrow 0$ найти значения $\varepsilon_{\infty}$ и $\varepsilon_{S}$ при различных температурах.

Шаг 2 предполагает нахождение параметра $\beta$. Используя тот факт, что в точке $\varepsilon_{S} \omega \rightarrow 0$ и соответственно $\Theta \rightarrow 0$, дифференцируя $\varepsilon^{\prime}$ и $\varepsilon^{\prime \prime}$, в пределе при $\Theta \rightarrow 0$ получаем

$$
\left|\left(\frac{d \varepsilon^{\prime \prime}}{d \varepsilon^{\prime}}\right) \varepsilon_{S}\right|=\frac{1}{\beta}
$$

Шаг 3 имеет своей целью определение параметра $\alpha$ в точке, соответствующей $\varepsilon_{\infty}$ при $\omega \rightarrow \infty$. В этой точке $\varepsilon^{\prime \prime}=\left(\varepsilon^{\prime}-\varepsilon\right) \operatorname{tg}(\beta \theta)$ и

$$
\begin{aligned}
\left(\frac{d \varepsilon^{\prime \prime}}{d \varepsilon^{\prime}}\right)_{\varepsilon_{\infty}} & =\operatorname{ctg}(\beta \theta) \\
& =\operatorname{ctg}\left[\beta \operatorname{arctg} \frac{(\omega \tau)^{1-\alpha} \cos \left(\frac{\alpha \pi}{2}\right)}{1+(\omega \tau)^{1-\alpha} \sin \left(\frac{\alpha \pi}{2}\right)}\right] .
\end{aligned}
$$

Учтя, что $\omega \rightarrow \infty$, имеем

$$
\begin{aligned}
\left(\frac{d \varepsilon^{\prime \prime}}{d \varepsilon^{\prime}}\right)_{\varepsilon_{\infty}} & =\operatorname{ctg}\left[\beta \operatorname{arctg} \frac{\cos \left(\frac{\alpha \pi}{2}\right)}{\frac{1}{(\omega \tau)^{1-\alpha}}+\sin \left(\frac{\alpha \pi}{2}\right)}\right] \\
& =\operatorname{ctg}\left[\beta \operatorname{arctg}\left(\operatorname{ctg}\left(\frac{\alpha \pi}{2}\right)\right)\right] .
\end{aligned}
$$

Учитывая, что $\operatorname{arctg}\left(\operatorname{ctg}\left(\frac{\alpha \pi}{2}\right)\right)=\frac{\pi}{2}-\frac{\alpha \pi}{2}$, после несложных преобразований получаем условие для нахождения параметра $\alpha$ :

$$
1-\alpha=\frac{\operatorname{arcctg}\left(\frac{d \varepsilon^{\prime \prime}}{d \varepsilon^{\prime}}\right)_{\varepsilon_{\infty}}}{\frac{\beta \pi}{2}} .
$$

Отметим, что вместо выбора $d \varepsilon^{\prime \prime} / d \varepsilon^{\prime}$ в качестве основы для нахождения дисперсионных параметров $\alpha$ и $\beta$ можно выбрать зависимость $\varepsilon^{\prime \prime}=f(\omega)$. Так, согласно [7],

$$
\left\{\begin{array}{l}
\lim _{\omega \rightarrow 0} \frac{d \lg \varepsilon^{\prime \prime}}{d \lg \omega}=1-\alpha \\
\lim _{\omega \rightarrow \infty} \frac{d \lg \varepsilon^{\prime \prime}}{d \lg \omega}=-(1-\alpha) \beta
\end{array}\right.
$$

Шаг 4 служит для нахождения последнего неизвестного параметра - времени релаксации $\tau$ при данной температуре. Это можно сделать двумя способами.
Первый способ основывается на соотношении, что для любой точки на диаграмме Арганда $\frac{\varepsilon^{\prime \prime}}{\varepsilon^{\prime}-\varepsilon_{\infty}}=\operatorname{tg}(\beta \theta)$. Тогда $\beta \theta=\operatorname{arctg}\left(\frac{\varepsilon^{\prime}}{\varepsilon^{\prime}-\varepsilon_{\infty}}\right)$ и

$$
\operatorname{arctg}\left[\frac{(\omega \tau)^{1-\alpha} \cos \left(\frac{\alpha \pi}{2}\right)}{1+(\omega \tau)^{1-\alpha} \sin \left(\frac{\alpha \pi}{2}\right)}\right]=\frac{1}{\beta} \operatorname{arctg}\left(\frac{\varepsilon^{\prime \prime}}{\varepsilon^{\prime}-\varepsilon_{\infty}}\right) .
$$

Основываясь на уравнениях (8) и (14), после преобразования получаем уравнение для времени релаксации:

$$
\tau=\frac{1}{\omega^{1-\alpha}} \sqrt{\frac{\operatorname{tg}\left(\left(\frac{1}{\beta}\right) \operatorname{arctg}\left(\frac{\varepsilon^{\prime \prime}}{\varepsilon^{\prime}-\varepsilon_{\infty}}\right)\right)}{\cos \left(\frac{\alpha \pi}{2}\right)-\sin \left(\frac{\alpha \pi}{2}\right)\left[\operatorname{tg}\left(\left(\frac{1}{\beta}\right) \operatorname{arctg}\left(\frac{\varepsilon^{\prime \prime}}{\varepsilon^{\prime}-\varepsilon_{\infty}}\right)\right)\right]}} .
$$

Для точности целесообразно решить задачу по крайней мере для трех точек, в одной из которых $\frac{d \varepsilon^{\prime \prime}}{d \varepsilon^{\prime}}=0$.

Способ 2 связан с положением точки, для которой $\omega \tau=1$. В этой точке

$$
\theta=\operatorname{arctg}\left[\frac{\cos \left(\frac{\alpha \pi}{2}\right)}{1+\sin \left(\frac{\alpha \pi}{2}\right)}\right]
$$

Используем (16)

$$
\begin{aligned}
\frac{\varepsilon^{\prime \prime}}{\varepsilon^{\prime}-\varepsilon_{\infty}} & =\operatorname{tg}(\beta \theta) \\
& =\operatorname{tg}\left(\beta \operatorname{arctg}\left[\frac{\cos \left(\frac{\alpha \pi}{2}\right)}{1+\sin \left(\frac{\alpha \pi}{2}\right)}\right]\right)=\operatorname{tg} \gamma .
\end{aligned}
$$

Рассчитав правую часть, т.е. $\operatorname{tg} \gamma$, получим уравнение прямой $\varepsilon^{\prime \prime}=\left(\varepsilon^{\prime}-\varepsilon_{\infty}\right) \operatorname{tg} \gamma$. Далее найдем точку пресечения с полиномиальным представлением $\varepsilon^{\prime \prime}=f\left(\varepsilon^{\prime}\right)$. Точка пересечения дает значение $\varepsilon^{\prime}$ и соответственно по частотной зависимости определяется частота $\omega$. Тогда определяем время релаксации $\tau=\frac{1}{\omega}$.

Шаг 5 предполагает варьирование параметров $\alpha, \beta$ и $\tau$ с целью нахождения наилучшего совпадения с экспериментальными величинами $\varepsilon^{\prime}$ и $\varepsilon^{\prime \prime}$. При этом можно использовать метод наименьших квадратов.

Описанная выше методика основана на методологии математического анализа. Вместе с тем компьютерные технологии в принципе позволяют организовать системно-интегральный подход для организации многофакторного анализа. Например, программный продукт WinFit, входящий в комплектацию широкополосного диэлектрического спектрометра BDS Novocontrol Concept 80 при условии установления системной GRID-платы. В работе [8] одним из соавторов предложена методика обработки на основе использования в версии программного обеспечения Origin 8.5 (Origin Lab Co) инструмента Fitting Function Builder, позволяющего программировать скрипты комплексных функций на языке C. 

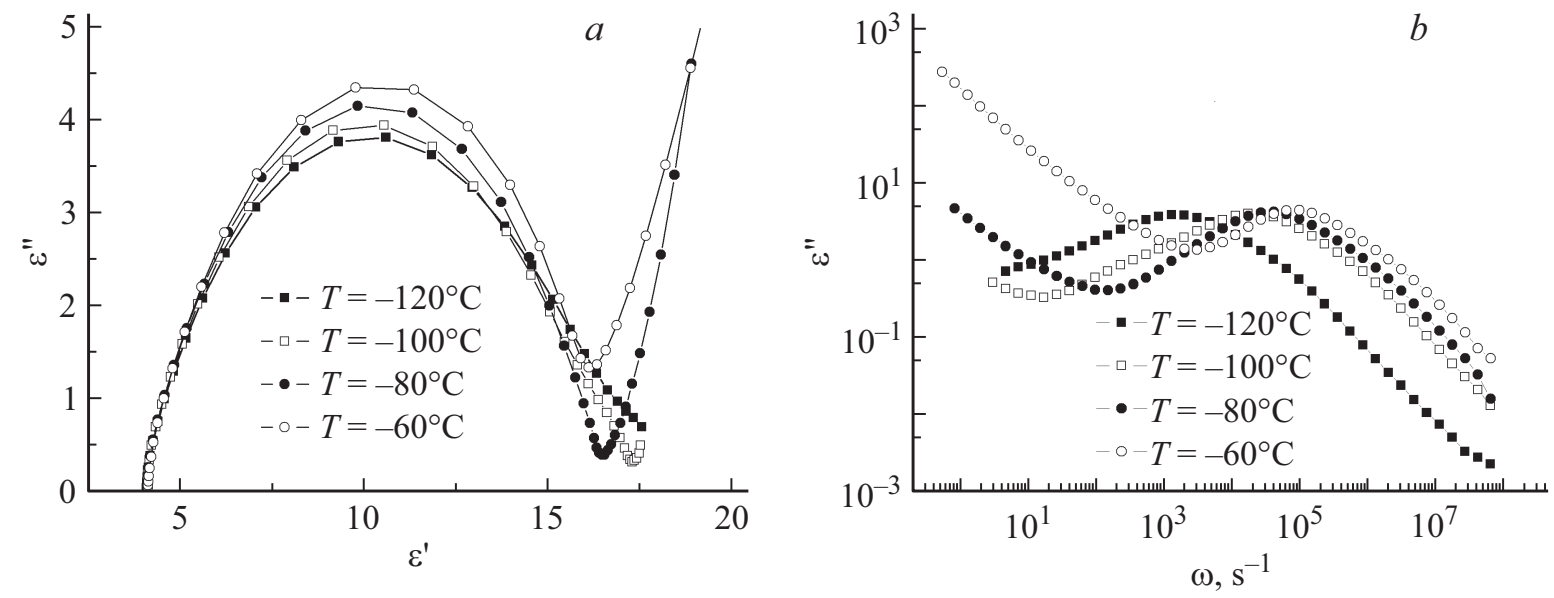

Рис. 1. Диаграммы Арганда $(a)$ и частотные зависимости $\varepsilon^{\prime \prime}(b)$ мерзлой (температура $\left.-60,-80,-100,-120^{\circ} \mathrm{C}\right)$ дисперсной среды при $W=13 \%$.
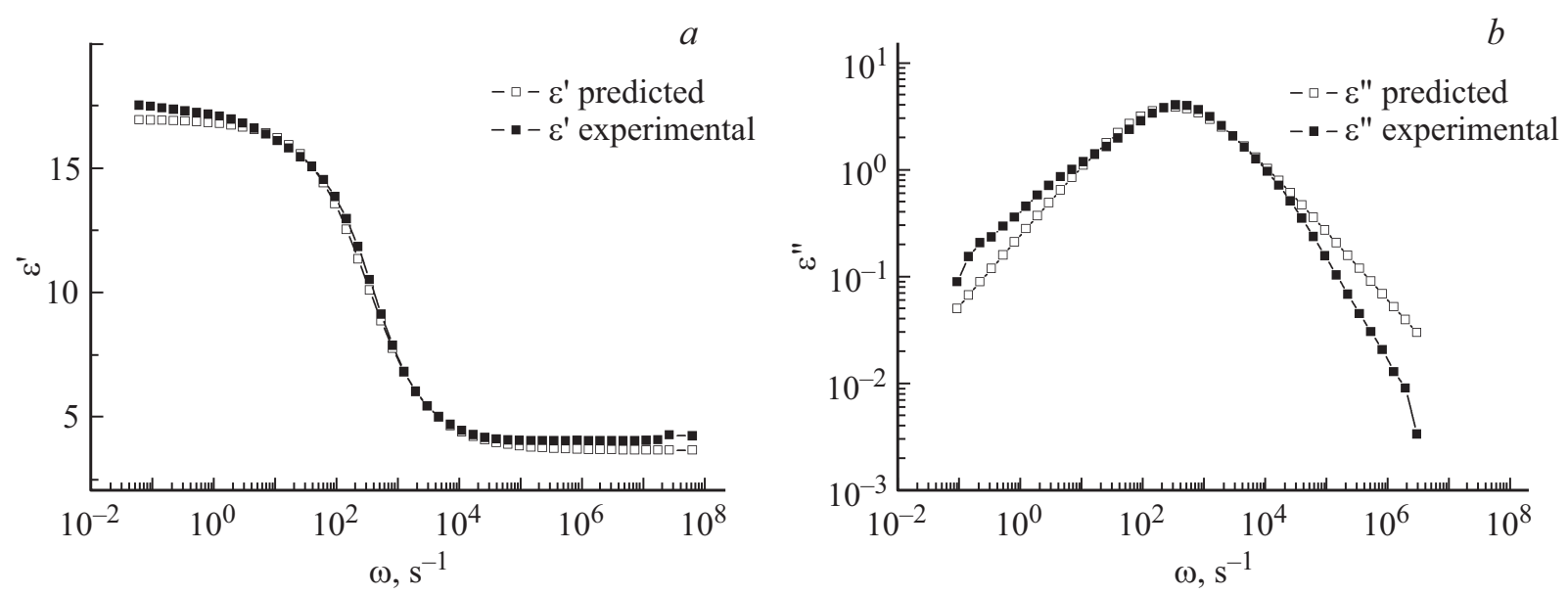

Рис. 2. Частотные зависимости $\varepsilon^{\prime}(a)$ и $\varepsilon^{\prime \prime}(b)$ мерзлой дисперсной среды при $W=13 \%$ и $t=-100^{\circ} \mathrm{C}, \varepsilon_{S}=16.93, \varepsilon_{\infty}=3.71$, $\alpha=0.33, \beta=0.96, \tau=3.3 \cdot 10^{-3} \mathrm{c}$.

\section{Экспериментальные результаты}

Авторы посчитали необходимым проиллюстрировать результаты, полученные при исследовании температурно-частотных зависимостей действительной и мнимой частей комплексной диэлектрической проницаемости мерзлой дисперсной среды на основе порошка кварца при влажности $13 \%$ в диапазоне температур от -140 до $0{ }^{\circ} \mathrm{C}$.

Исследование проводилось на широкополосном диэлектрическом спектрометре BDS Novocontrol Concept 80 в диапазоне частот от $10^{-2}$ до $10^{6}$ Гц и диапазоне температур от -140 до $0^{\circ} \mathrm{C}$. Диаграммы Арганда и зависимости $\varepsilon^{\prime \prime}$ от $\omega$ представлены на рис. 1. Результаты определения параметров частотной дисперсии представлены в таблице.

Отметим, что проведенный анализ справедлив для любых значений $\varepsilon_{S}-\varepsilon_{\infty}$, так как $\frac{\varepsilon^{\prime \prime}}{\varepsilon_{S}-\varepsilon_{\infty}}$ не зависит от $\varepsilon_{S}-\varepsilon_{\infty}$, а определяется параметрами $\alpha, \beta$ и $\tau$ на любой частоте.
Для подтверждения правильности полученных результатов по определению параметров в модели ГаврилякаНегами на рис. 2 приведены графики зависимостей $\varepsilon^{\prime}$ и $\varepsilon^{\prime \prime}$ от частоты $\omega$.

Слабым местом проведенного анализа является использование значений $\varepsilon^{\prime \prime}$, точность измерения которых обычно ниже, чем точность измерения $\varepsilon^{\prime}$. Но использование диэлектрического спектрометра BDS Novocontrol Concept 80 позволяет снизить погрешности при измерениях как $\varepsilon^{\prime \prime}$, так и $\varepsilon^{\prime}$.

Параметры частотной дисперсии в модели Гавриляка-Негами для мерзлой дисперсной среды на основе порошка кварца влажностью $13 \%$

\begin{tabular}{c|c|c|c|c|c}
\hline$t, \mathrm{~K}$ & $\varepsilon_{S}$ & $\varepsilon_{\infty}$ & $\alpha$ & $\beta$ & $\tau, \mathrm{c}$ \\
\hline 153 & 13.04 & 0.95 & 0.30 & 0.95 & $3.03 \cdot 10^{-2}$ \\
173 & 12.94 & 1.118 & 0.24 & 0.95 & $2.50 \cdot 10^{-3}$ \\
193 & 13.015 & 1.246 & 0.24 & 0.98 & $6.49 \cdot 10^{-4}$ \\
213 & 13.09 & 1.243 & 0.26 & 0.99 & $2.70 \cdot 10^{-4}$
\end{tabular}




\section{Список литературы}

[1] Дебай П. Полярные молекулы. М.-Л.: ГНТИ, 1931. 241 с.

[2] Cole K.S., Cole R.H. // J. Phys. Chem. 1941. V. 9. P. 341.

[3] Devidson D.W., Cole R.H. // J. Chem. Phys. 1951. V. 19. P. 1484.

[4] Havriliak S., Negami S. // J. Polym. Sci. C. 1966. V. 14. P. 99.

[5] Челидзе T.Л. Электрическая спектроскопия гетерогенных систем. Киев: Наукова думка, 1977. 231 с.

[6] Лукичёв А.А. // Известия Самарского научного центра РАН. 2015. T. 15. № 4. C. 35.

[7] Андреев Е.В., Богатин А.С., Ковригина С.А., Игнатова Ю.А., Богатина В.Н., Носачев И.О. // Фазовые переходы, межфазные границы и наноматериалы, 2015. № 1. C. 134.

[8] Волков А.С., Волкова Ю.В. // Физический вестник Института естественных наук и технологий САФУ. Вып. 14. Архангельск: Кира, 2015. С. 119 